\title{
DEMANDAS DE ATENDIMENTO DE ENFERMAGEM E DE QUALIFICAÇÃO EM ONCOLOGIA NA ATENÇÃO BÁSICA EM SAÚDE
}

\author{
Luciana Martins da Rosa ${ }^{1}$, Ana Izabel Jatobá de Souza ${ }^{1}$, Jane Cristina Anders ${ }^{1}$, Rafaela Nunes da Silva ${ }^{2}$, \\ Gabriela Schutz da Silva ${ }^{3}$, Mayara Cristine Fontão
}

\begin{abstract}
RESUMO: O objetivo deste estudo foi identificar as demandas de atendimento e de qualificação em Oncologia dos enfermeiros atuantes na Atenção Básica. Estudo observacional, descritivo, realizado nos Centros de Saúde de Florianópolis, Santa Catarina, Brasil. A coleta de dados ocorreu entre outubro e novembro de 2015. Investigou variáveis relacionadas à formação, tempo de atuação profissional, demanda de atendimento e déficits de qualificação em oncologia. Participaram do estudo 84 enfermeiros; 41 (48,81\%) formados há mais de dez anos $(12,04 \pm 6,99) ; 46(54,76 \%)$ consideram insuficiente o aprendizado sobre oncologia na graduação e referem ausência de aperfeiçoamento nesta área após a formação. As principais demandas de atendimento e de déficit de qualificação envolvem as neoplasias malignas mais incidentes, o impacto do câncer, as terapêuticas oncológicas e seus efeitos colaterais. Os dados evidenciam que, apesar da experiência na atenção básica, muitos profissionais sentem a necessidade de capacitações que os habilitem ao atendimento especializado e qualificado.

DESCRITORES: Enfermagem; Oncologia; Capacitação profissional; Atenção primária à saúde.
\end{abstract}

\section{ONCOLOGY NURSING CARE AND QUALIFICATION DEMANDS IN PRIMARY HEALTH CARE}

ABSTRACT: The objective in this study was to identify the oncology care and qualification demands of nurses working in primary health care. Observational and descriptive study developed at the Health Centers in Florianópolis, Santa Catarina, Brazil. The data were collected between October and November 2015. The research variables were related to education, length of professional experience, care demand and qualification deficiencies in oncology. The study participants were 84 nurses; 41 (48.81\%) had graduated more than ten years earlier (12.04 \pm 6.99$) ; 46(54.76 \%)$ consider that their learning about oncology at the undergraduate level was insufficient and indicate no further qualification in this area after graduating. The main care and qualification deficiency demands involve the most incident malign tumors, the impact of cancer, oncology therapeutics and their side effects. The data evidence that, despite the experience in primary health care, many professionals feel the need for training that enables them to deliver specialized and qualified care.

DESCRIPTORS: Nursing; Oncology; Professional education; Primary health care.

\section{DEMANDAS DE ATENCIÓN DE ENFERMERÍA Y DE CUALIFICACIÓN EN ONCOLOGÍA EN LA ATENCIÓN BÁSICA DE SALUD}

RESUMEN: El objetivo de este estudio fue identificar las demandas de atención y de cualificación en Oncología de los enfermeros que actúen en la Atención Básica. Estudio observacional, descriptivo, desarrollado en los Centros de Salud de Florianópolis, Santa Catarina, Brasil. Los datos fueron recolectados entre octubre y noviembre del 2015. Investigó variables relacionadas a la formación, tiempo de actuación profesional, demanda de atención y déficits de cualificación en oncología. Participaron del estudio 84 enfermeros; $41(48,81 \%)$ formados desde hace más de diez años $(12,04 \pm 6,99) ; 46(54,76 \%)$ consideran insuficiente el aprendizaje sobre oncología en el pregrado y refieren ausencia de perfeccionamiento en esta área tras la formación. Las principales demandas de atención y de déficit de cualificación implican las neoplasias malignas más incidentes, el impacto del cáncer, las terapéuticas oncológicas y sus efectos colaterales. Los datos evidencian que, a pesar de la experiencia en la atención básica, muchos profesionales sienten la necesidad de capacitaciones que les habiliten a la atención especializada y cualificada.

DESCRIPTORES: Enfermería; Oncología; Capacitación profesional; Atención primaria de salud.

${ }^{1}$ Enfermeira. Doutora em Enfermagem. Docente de Enfermagem e de Pós-Graduação em Gestão do Cuidado em Enfermagem da Universidade Federal de Santa Catarina. Florianópolis, SC, Brasil.

${ }^{2}$ Enfermeira. Instituto da Visão Assad Rayes. Florianópolis, SC, Brasil.

${ }^{3}$ Enfermeira. Universidade Federal de Santa Catarina. Florianópolis, SC, Brasil.

${ }^{4}$ Enfermeira. Clínica Core. Florianópolis, SC, Brasil. 


\section{INTRODUÇÃO}

Nas últimas décadas, o câncer ganhou relevância mundial, dadas as evidências do perfil epidemiológico no Brasil e no mundo. Sendo assim, vem sendo considerado um problema de saúde pública. As transformações demográficas advindas do envelhecimento populacional, a industrialização, a urbanização, os avanços da ciência e da tecnologia, os novos estilos de vida e de exposição aos fatores de risco para o câncer estão elevando as taxas de morbimortalidade ocasionadas pela doença e preocupando os governantes e a sociedade ${ }^{(1)}$.

O Instituto Nacional do Câncer (INCA) afirma que a prevenção e o controle do câncer precisam adquirir o mesmo foco e a mesma atenção dispensada à área de serviços assistenciais, pois o crescente aumento do número de casos novos de câncer fará com que, nas próximas décadas, o sistema de saúde pública não tenha recursos financeiros e humanos suficientes para o atendimento da demanda oncológica no país. Esta condição poderá contribuir para mortes prematuras e desnecessárias ${ }^{(1)}$.

A International Agency for Research on Cancer (IARC), vinculada à Organização Mundial da Saúde (OMS), afirma a incidência de 14,1 milhões de casos novos de câncer e um total de 8,2 milhões de mortes a cada ano em todo o mundo. Para 2030 a IARC afirma que ocorrerão no mundo cerca de 21,4 milhões de casos novos de câncer e 13,2 milhões de mortes por câncer ${ }^{(2)}$. O INCA estima que ocorrerão no Brasil no ano de 2016/2017 cerca de 600 mil casos novos de câncer, o que reforça a magnitude do problema do câncer no país ${ }^{(1)}$.

Em relação à formação e especialização dos recursos humanos no atendimento às pessoas com suspeita, diagnóstico ou sobrevivente ao câncer, observa-se lacuna considerável relacionada à capacitação da enfermagem para as demandas oncológicas no processo de viver e de morrer, lacuna esta que se inicia na graduação. A maioria dos cursos de graduação em Enfermagem existentes no Brasil não aborda com aprofundamento temas relacionadas à oncologia e a enfermagem oncológica ${ }^{(3-6)}$, mesmo sendo o câncer um expressivo problema de saúde pública e com preocupantes estimativas para o futuro, como já apontadas anteriormente.

Um estudo identificou as demandas de qualificação em oncologia para as diferentes categorias profissionais da área da saúde, nas diversas regiões do país. Os resultados apontaram que os temas prioritários para qualificação na demanda nacional foram: atuação profissional ao paciente oncológico na atenção básica $(65,8 \%)$, seguida da atuação ambulatorial $(64,4 \%)$, noções básicas de controle e prevenção ao câncer $(63,7 \%)$, atuação profissional ao paciente oncológico no contexto hospitalar $(61,6 \%)$, e em cuidados paliativos $(61,2 \%)^{(7)}$. Na investigação junto aos enfermeiros, o referido estudo identificou que os temas de maior necessidade para qualificação foram: noções básicas de controle e prevenção do câncer $(71,5 \%)$, atuação profissional na promoção à saúde e na prevenção de agravos direcionados à oncologia (69,4\%), atuação profissional ao paciente oncológico ambulatorial $(67,2 \%)$, gestão e políticas públicas de atenção ao câncer $(66,7 \%)^{(7-8)}$.

Este ainda apontou que os enfermeiros referiram a crescente inserção dos profissionais na atenção básica como justificativa para uma qualificação maior em oncologia, tendo em vista os potenciais benefícios que isso poderia trazer à população. Neste contexto, mencionaram a carência de enfermeiros especialistas em oncologia. Destaca-se que foi mencionada a necessidade de qualificação da enfermagem em toda a linha de cuidado, desde os procedimentos de menor complexidade até os mais complexos ${ }^{(7-8)}$.

Os achados mencionados levam à reflexão sobre a necessidade de qualificação dos enfermeiros para o atendimento dos usuários do Sistema Único de Saúde (SUS) com diagnóstico de câncer. Portanto, o presente estudo tem o seguinte questionamento: quais são as demandas de qualificação em oncologia observadas pelos enfermeiros atuantes na atenção básica no município de Florianópolis, estado de Santa Catarina? E como objetivo: identificar as demandas de qualificação em oncologia dos enfermeiros atuantes na atenção básica no município de Florianópolis-SC.

Dada a incidência e prevalência da doença encontrada nos dias atuais e a magnitude esperada para um futuro próximo, percebe-se a relevância deste estudo a fim de aprimorar a qualificação dos profissionais de enfermagem da atenção básica, abordando os cuidados relacionados às pessoas com câncer. $E$, para que isso aconteça, é preciso primeiramente a identificação das demandas de 
qualificação desta categoria profissional, para que se possam criar estratégias para aprimoramento de tal qualificação. Ressalta-se que a atenção básica está diretamente relacionada às novas políticas públicas que destacam a promoção da saúde e o atendimento, utilizando-se os diversos serviços ofertados pela rede de atenção SUS.

Os resultados deste estudo contribuirão para a elaboração de um ambiente virtual de aprendizagem para os profissionais do cenário do estudo, contendo as demandas de qualificação apontadas pelos mesmos, ampliando a consolidação de estratégias vinculadas à educação permanente.

\section{- MÉTODO}

Estudo descritivo realizado nos Distritos Sanitários de Saúde do município de Florianópolis-SC. Foram incluídos no estudo enfermeiros atuantes nos Centros de Saúde dos Distritos Sanitários: Centro, Continente, Leste, Norte e Sul (55 Centros de Saúde). Estes Distritos contam com a atuação de 162 enfermeiros (população do estudo).

A amostragem estabelecida, com erro amostral de $10 \%$ e nível de confiança $99 \%$, foi de 83 enfermeiros, porém foram incluídos no estudo 84 enfermeiros. O cálculo amostral foi realizado para definição de amostra mínima necessária, mas todos os enfermeiros atuantes no cenário do estudo foram convidados para inclusão no estudo.

O critério de inclusão definido para seleção dos participantes consistiu em ser enfermeiro atuante no cenário do estudo, independente do tempo de atuação. As exclusões se deram por afastamento de suas funções por motivo de férias, atestados, licenças ou similares no momento da coleta de dados (cerca de 50 profissionais). Foram também excluídos enfermeiros residentes. Cerca de 28 profissionais optaram pela não inclusão no estudo.

A coleta de dados foi realizada nos meses de outubro e novembro de 2015, durante as reuniões mensais dos enfermeiros em cada Distrito Sanitário, por meio de aplicação de questionário, contendo perguntas abertas e fechadas. As variáveis do estudo investigaram dados relacionados à formação, tempo de experiência profissional na atenção básica e oncológica, demandas de atendimento ao paciente oncológico, déficits de qualificação em oncologia para o atendimento do usuário do Sistema Único de Saúde (SUS), seja criança, adolescente e/ou adulto, com suspeita ou com diagnóstico confirmado de câncer e para o sobrevivente do câncer na atenção básica em Florianópolis-SC.

Os dados coletados foram registrados em planilhas construídas no programa Excel da Microsoft, agrupados por similaridade e submetidos à análise estatística descritiva (medidas de frequência, média e desvio padrão), depois discutida à luz da literatura científica sobre a temática.

O estudo foi desenvolvido de acordo com as determinações legais para pesquisa com seres humanos e foi aprovado por Comitê de Ética em Pesquisa, parecer número 1.238.414. O anonimato dos participantes foi garantido mediante a entrega dos questionários sem identificação dos participantes.

\section{RESULTADOS}

Atuavam nos Distritos Sanitários de Saúde, cenário deste estudo, 162 (100\%) enfermeiros, e aceitaram ser participantes deste estudo 84 enfermeiros (51,85\%).

Dos 84 (100\%) profissionais incluídos no estudo, 23 (27,38\%) atuavam no Distrito Centro, 21 (25\%) Distrito Continente, 15 (17,86\%) Distrito Leste, nove (10,71\%) Distrito Sul e 16 (19,05\%) no Distrito Norte.

Quanto ao tempo de formação, um (1,19\%) enfermeiro estava formado há um ano; nove (10,71\%) entre 1 e 5 anos; 33 (39,28\%) entre 6 e 10 anos; 41 (48,81\%) há mais de 10 anos (12,04 $\pm 6,99)$. Quanto ao tempo de atuação na atenção básica, cinco (5,95\%) tinham experiência de até 1 ano; 26 (30,95\%) entre 1 e 5 anos; 27 (32,14\%) entre 6 e 10 anos; e 26 (30,95\%) mais de 10 anos $(6,62 \pm 6,05)$.

Em relação ao tempo de experiência no cuidado de enfermagem na oncologia fora da atenção básica, 73 participantes (86,9\%) registraram não ter experiência, seis $(7,14 \%)$ registraram até um ano de 
experiência, três $(3,57 \%)$ entre um e cinco anos e dois $(2,38 \%)$ com seis anos ou mais $(8,62 \pm 6,05)$.

A maioria dos enfermeiros, 52 (61,9\%), é egressa do Curso de Graduação em Enfermagem da Universidade Federal de Santa Catarina (UFSC), oito (9,52\%) da Universidade do Vale do Itajaí (UNIVALI), quatro (4,76\%) da Universidade do Sul de Santa Catarina (UNISUL), 16 (19,05\%) de outras instituições de ensino do Estado de Santa Catarina, Rio Grande do Sul e Paraná, um (1,19\%) do Estado da Bahia, três $(3,57 \%)$ não registraram.

A avaliação dos enfermeiros frente aos conteúdos teóricos e práticos sobre a oncologia aprendidos na graduação em enfermagem equivaleu a: nenhum participante considerou excelente, oito $(9,53 \%)$ consideraram bom, 30 (35,71\%) regular e 46 (54,76\%) insuficiente.

O aperfeiçoamento em oncologia foi declarado por três enfermeiros (3,57\%). Os aperfeiçoamentos citados foram: Curso de Especialização em Enfermagem Oncológica em Sistemas de Rede promovido pela Universidade Federal de Santa Catarina e o Curso ABC do Câncer disponibilizado a distância pelo INCA.

Quando questionados sobre as demandas de atendimento em oncologia que os enfermeiros atuantes nos Centros de Saúde se deparam, quatro participantes (4,76\%) optaram por não responder. As respostas registradas nos questionários foram agrupadas em 21 demandas. Destas demandas as cinco mais citadas foram: neoplasias malignas: câncer de mama, de útero, de intestino, de próstata, de pulmão, de tireoide, de pele, de cabeça e pescoço, de esôfago, hepático, cânceres associados às comorbidades, leucemia, linfoma; impacto do câncer na criança, adolescente, adulto e família; tratamento quimioterápico e pós-quimioterapia; cuidados após o término da terapêutica oncológica; exames no controle do câncer. Na Tabela 1 apresentam-se todas as demandas de atendimento no contexto da oncologia no cenário do estudo.

Tabela 1 - Demandas de atendimento ao usuário do SUS na atenção básica segundo enfermeiros. Florianópolis, SC, Brasil, 2015

\section{Demandas de atendimento}

Neoplasias malignas: câncer de mama (19), de útero (13), de intestino (11), de próstata (4),

de pulmão (4), de tireoide (4), de pele (3), de cabeça e pescoço (2), de esôfago (1), hepático

(1), cânceres associados às comorbidades (2), leucemia (4), linfoma (1)**

\begin{tabular}{|c|c|c|}
\hline Impacto do câncer na criança, adolescente, adulto e família & 12 & 6,38 \\
\hline Cuidados após o término da terapêutica oncológica & 11 & 5,85 \\
\hline Exames para o controle do câncer & 11 & 5,85 \\
\hline Tratamento quimioterápico (7) e pós-quimioterapia (3)** & 10 & 5,31 \\
\hline Procedimentos de enfermagem & 8 & 4,25 \\
\hline Cuidados com estomas & 8 & 4,25 \\
\hline Fase pré (2) e pós-operatória (6)** & 8 & 4,25 \\
\hline Cuidados paliativos. & 7 & 3,72 \\
\hline Encaminhamentos na Rede de Atenção à Saúde & 7 & 3,72 \\
\hline Efeitos colaterais das terapêuticas oncológicas & 6 & 3,19 \\
\hline Fase de diagnóstico do câncer & 4 & 2,12 \\
\hline Fase pós-alta do tratamento oncológico & 4 & 2,12 \\
\hline Direitos dos usuários oncológicos & 4 & 2,12 \\
\hline Visita domiciliar & 4 & 2,12 \\
\hline Tratamento radioterápico e cuidados pós-radioterapia & 4 & 2,12 \\
\hline Prevenção do câncer & 3 & 1,59 \\
\hline Qualidade de vida & 2 & 1,06 \\
\hline Dor aguda na pessoa com câncer & 2 & 1,06 \\
\hline Disponibilização e orientações - receituário médico oncologista & 2 & 1,06 \\
\hline Fornecimento e orientações para uso de medicamentos prescritos & 2 & 1,06 \\
\hline Total & 188 & 100 \\
\hline
\end{tabular}


Fonte: Dados da pesquisa.

* Frequência da citação da demanda por todos os enfermeiros incluídos no estudo.

**Para facilitar a apresentação das demandas, algumas delas foram agrupadas, porém a frequência absoluta foi registrada por item citado.

Em relação às demandas de qualificação em oncologia (déficits de qualificação), os participantes elencaram 23 demandas que foram agrupadas por similaridade. Vale ressaltar que um participante poderia sugerir mais de uma demanda, destas, as cinco mais citadas foram: neoplasias malignas e cuidados relacionados - câncer de mama, de útero, de pulmão, de fígado, de próstata, metástases, recidivas do câncer e cuidados especializados por tipo de câncer; terapêuticas no controle do câncer; efeitos colaterais ocasionados pelas terapêuticas oncológicas; impacto do câncer na criança, adolescente, adulto e família; e rede de atenção e suporte ao paciente oncológico. Na Tabela 2 apresenta-se a totalidade dos achados.

Tabela 2 - Demandas/déficits de qualificação no atendimento ao usuário do SUS na Atenção Básica segundo enfermeiros. Florianópolis, SC, Brasil, 2015

\begin{tabular}{llc} 
Demandas/déficits de qualificação & n* & \% \\
\hline $\begin{array}{l}\text { Neoplasias malignas e cuidados relacionados: câncer de mama(4), câncer de útero (3), } \\
\text { câncer de pulmão (1), câncer de fígado (1), câncer de próstata (1), metástases (1), recidivas } \\
\text { do câncer (1) e cuidados especializados por tipo de câncer (12)** }\end{array}$ & 24,81 \\
\hline Terapêuticas no controle do câncer & 20 & 12,34 \\
\hline Efeitos colaterais ocasionados pelas terapêuticas oncológicas & 18 & 11,11 \\
\hline Impacto do câncer na criança, adolescente, adulto e família & 17 & 10,49 \\
\hline Rede de atenção e suporte ao paciente oncológico & 15 & 9,25 \\
\hline Diagnóstico (6) e prognóstico do câncer (2)** & 8 & 4,93 \\
\hline Educação permanente no contexto da oncologia & 3,7 \\
\hline Cuidados paliativos ao paciente oncológico & 6 & 3,7 \\
\hline Direitos do paciente oncológico & 6 & 3,7 \\
\hline Farmacoterapia oncológica & 6 & 3,08 \\
\hline Funcionamento e rotinas do CEPON - Centro de Pesquisas Oncológicas & 3,08 \\
\hline Cuidado com feridas (2), estomas (2) e traqueostomias (1)** & 5 & 3,08 \\
\hline Exames de controle e suas alterações & 5 & 2,46 \\
\hline Sinais e sintomas do câncer e o diagnóstico precoce & 5 & 2,46 \\
\hline Cuidado no pós-operatório (2) e pós-mastectomia (2)** & 4 & 2,46 \\
\hline Prevenção (1) e rastreamento (3) do câncer** & 4 & 2,46 \\
\hline Cuidados domiciliares & 4 & 4 \\
\hline Procedimentos de enfermagem & 4 & 1,85 \\
\hline Estenose vaginal & 3 & 1,23 \\
\hline Qualidade de vida & 2 & 1,23 \\
\hline Cuidados com cateter semi-implantado & 2 & 1 \\
\hline Cuidados por faixa etária & 1 & 0,61 \\
\hline Alimentação no contexto do câncer & 0,61 \\
\hline Total & 0,61 \\
\hline & 0,61 \\
\hline & 100
\end{tabular}

Fonte: Dados da pesquisa.

*Frequência da citação da demanda por todos os enfermeiros incluídos no estudo.

**Para facilitar a apresentação das demandas, algumas delas foram agrupadas, porém a frequência absoluta foi registrada por item citado. 


\section{- DISCUSSÃO}

Quanto ao tempo de formação e tempo de experiência na atenção básica, observou-se que a maioria dos participantes tem experiência no referido contexto. A relação entre o tempo de formação, tempo de experiência profissional e o tempo de experiência no mesmo contexto de trabalho dá ao trabalhador maior competência para o exercício profissional, consequentemente, melhores possibilidades para tomadas de decisão e melhores resultados a serem atingidos, neste caso, às pessoas com necessidades básicas em saúde.

A graduação em enfermagem revela competências e habilidades do enfermeiro que se consolidam pela aprendizagem continuada, abrangendo um conjunto de aprendizagens sociais e comunicacionais. É um atributo conquistado por ações individuais para aquisição e construção de conhecimentos de um sujeito, mas que deve ter como base as demandas das situações concretas do trabalho que envolvem a construção pautada em bases socioculturais, históricas e de educação permanente. Um profissional competente sabe agir com responsabilidade, é reconhecido pelos seus pares; sabe como mobilizar, integrar e "transferir" seus conhecimentos e habilidades, num contexto profissional determinado ${ }^{(9-10)}$.

Entretanto, apesar do tempo de experiência profissional dos participantes deste estudo, eles mesmos revelam a carência e/ou insuficiência de formação e as necessidades de qualificação para o atendimento de adultos, crianças, adolescentes e sobreviventes com câncer ou com suspeita da doença. Esta carência caracteriza-se por déficit da formação e das práticas de educação permanente, pois a grande maioria dos participantes aponta tal condição.

O INCA, órgão norteador das ações oncológicas no Brasil, também afirma a carência de formação dos profissionais para o cuidado relacionado à atenção oncológica para todas as categorias profissionais, e que os déficits de qualificação se destacam na atenção básica de saúde ${ }^{(7)}$.

Então, como alcançar a competência necessária (conhecimento, habilidade e atitude) diante de um cenário de carência de formação e de educação permanente? Como tomar decisões acertadas? Como contribuir para a melhoria da atenção à saúde pensando na eficácia do sistema público de saúde (qualidade do atendimento, melhores resultados com menores custos possíveis)?

As melhores tomadas de decisões no cuidado à saúde, dentre outros aspectos, envolvem: a avaliação das reais necessidades dos usuários; a realização do trabalho pautado em planejamento que contemple o detalhamento de informações; o pensamento crítico (adquirido por formação competente e experiência profissional) sobre as situações, com base em análise e julgamento das perspectivas de cada proposta de ação e de seus desdobramentos; o raciocínio lógico e intuitivo; a avaliação do processo; o desenvolvimento da cultura institucional para atendimento das necessidades da população; e, a revisão dos modelos de comportamento ${ }^{(11)}$.

Para tanto, as atividades de educação permanente, conforme orientações do Ministério da Saúde, podem assegurar a manutenção da competência da equipe de enfermagem ${ }^{(11)}$ em relação ao cuidado dos usuários do SUS na atenção básica e no contexto da Oncologia.

Além da educação permanente, é crucial a mudança nos currículos de formação para atendimento das necessidades da população brasileira. Os currículos precisam aproximar a teoria da prática e das necessidades sociais, pois o câncer é uma das doenças mais incidentes e que mais leva ao óbito no território nacional e no mundo.

E ainda, as estimativas do câncer tendem a se elevar nas próximas décadas. Igualmente, nesta demanda inclui-se um número maior de sobreviventes, de pessoas em recidiva da doença e em cuidados paliativos, pois com o desenvolvimento tecnológico, o controle da doença está cada vez maior. Entretanto, a cura ainda não foi alcançada na mesma proporção. Assim, a sobrevivência do câncer traz as necessidades de cuidados dos profissionais da área da saúde, de forma que possam atender às pessoas em qualquer fase da doença e condição de saúde.

Atualmente, a maior parcela de atendimento do paciente oncológico ainda se dá na alta complexidade, mas a mudança demográfica e epidemiológica e a necessidade da equidade e integralidade na saúde pública tornam necessário que o atendimento em rede de atenção realmente se efetive. Este é uma 
prerrogativa do Sistema Único de Saúde (SUS) e que precisa ser efetivada articulando os pontos da rede em benefício da população.

O mercado de trabalho em saúde vem se transformando pelas políticas econômicas, tecnológicas e sociais, e essas transformações exigem reformulações urgentes das instituições de ensino, formadores dos profissionais da área da saúde. Somente assim os egressos atenderão às novas demandas e resolução de problemas. O desafio das instituições de ensino superior de enfermagem deve ser o de preparar enfermeiros com competência técnica e política, como sujeitos sociais dotados de conhecimento, de raciocínio para as questões da vida e da sociedade, capacitando-os para a melhor intervenção( ${ }^{(9)}$.

Considerando que a maioria dos participantes deste estudo é egressa da UFSC, UNIVALI e UNISUL, evidencia-se que estas instituições de ensino devem adequar o conteúdo curricular para que os profissionais enfermeiros possam, ao se formar, sentir-se aptos ao cuidado mínimo à pessoa com câncer ou suspeita da doença em todos os níveis de atenção no SUS.

Estende-se isto como um compromisso social e de retorno aos usuários, visto que a maioria dos participantes concluiu o curso de graduação em enfermagem em uma instituição pública. Cabe às instituições de ensino superior público dar retorno à sociedade, que subsidia sua existência. Há décadas o câncer é um problema de saúde pública, que atinge o indivíduo, sua família e a própria sociedade. Ao constatarmos as inúmeras mortes prematuras e incapacidades decorrentes da doença, torna-se urgente a mudança no processo de formação em articulação com as mudanças das políticas públicas estabelecidas nos últimos anos, que evidenciam a promoção da saúde, o atendimento em rede de atenção e a competência profissional.

No que se refere às demandas de atendimento, percebe-se que as mais prevalentes estão relacionadas às patologias mais incidentes no âmbito oncológico, seguida de constatação de que esta situação traz impacto sobre o indivíduo e família. Portanto, abordar o impacto do câncer no indivíduo e na família pode ser uma fragilidade dos profissionais, uma vez que neste devem estar contido conteúdos significativos voltados aos aspectos psicoemocionais relacionados à doença oncológica. É importante destacar que nem todos os profissionais da saúde se sentem capacitados para trabalhar algumas das dimensões psicoemocionais envolvidas no adoecimento crônico, em especial quando se fala em câncer, que por si só traz consigo conotações de finitude antecipada e sofrimento.

No contexto investigado, destacou-se a grande demanda vinculada aos cânceres mais prevalentes, confirmando as tendências epidemiológicas dos indicadores veiculados pelo Ministério da Saúde e órgãos internacionais. Capacitar para o entendimento dos cânceres mais prevalentes, atrelado ao impacto que desencadeiam no indivíduo e na família, igualmente se reflete nos déficits de qualificação, evidenciada pelos participantes do estudo.

No âmbito dos déficits de qualificação, é possível perceber a convergência entre os principais atendimentos e a necessidade de ser capacitado para eles. Nos déficits de qualificação encontram-se dimensões técnicas bem delineadas, tais como cuidados com feridas, com o uso de traqueostomias, entre outros. Contudo, os dados encontrados mostram a necessidade de capacitação para a compreensão dos cânceres mais prevalentes, suas formas de tratamento e de abordagem psicoemocional relacionado ao impacto dos mesmos, ou seja, as principais demandas de atendimento também são as principais demandas de qualificação.

Evidenciou-se também a necessidade de informação por parte dos participantes do estudo, no que se refere à interlocução com a instituição que atende os usuários do SUS com câncer que é, prioritariamente, o CEPON. Compreender o fluxo de atendimento do usuário com câncer e rede de atenção do SUS é crucial para todos os profissionais e para a qualidade da atenção.

A incidência e prevalência do câncer exigem um maior nível de conhecimento dos profissionais da área da saúde, nos diversos níveis de atenção à saúde no âmbito do SUS, justificando a necessidade de capacitações e educação permanente para todos os enfermeiros, desde os que atuam em unidades hospitalares exclusivamente oncológicas, até os que atuam na atenção básica. A falta de conhecimento dos profissionais pode acarretar prejuízos aos pacientes oncológicos ${ }^{(12)}$, por outro lado, o estreitamento das relações entre as instituições de ensino e de saúde pode contribuir para o aperfeiçoamento técnico e científico dos enfermeiros ${ }^{(13)}$. 
Os resultados deste estudo mostram a realidade de enfermeiros que atuam na atenção básica de saúde e que atendem usuários com diagnóstico e em tratamento oncológico, evidenciando importantes demandas de atendimento e de déficits de conhecimento que podem contribuir para a qualificação do atendimento. Estas principais demandas incluem os cânceres mais incidentes, o impacto do câncer, terapêuticas oncológicas e os cuidados de enfermagem relacionados, e a rede de atenção e suporte ao paciente oncológico.

Os resultados ainda revelam que os enfermeiros, mesmo atuando há algum tempo na atenção básica, sentem falta de capacitações que os habilite a proporcionar um atendimento ainda mais qualificado. Assim, foi possível perceber que os órgãos formadores destes profissionais ainda não trazem conhecimentos sobre o câncer e todos os seus desdobramentos em sua formação curricular. Daí a ausência de formação que proporcione maior segurança na abordagem das questões oncológicas trazidas pelos pacientes. Tais aspectos desembocam nas necessidades de qualificação, apontadas pelos participantes deste estudo, que devem ser minimizadas pela educação permanente.

Como limite do estudo, registra-se o uso de perguntas abertas exclusivas para coleta de dados para identificação dos déficits de qualificação. Entende-se que o uso de perguntas abertas e fechadas poderia ter ampliado os resultados do estudo.

\section{REFERÊNCIAS}

1. Instituto Nacional de Câncer José Alencar Gomes da Silva (INCA). Coordenação de Prevenção e Vigilância. Estimativa 2016: incidência de câncer no Brasil. [Internet] Rio de Janeiro: INCA; 2015 [acesso em 19 mai 2017]. Disponível: http://www.inca.gov.br/estimativa/2016/.

2. World Health Organization (WHO). International Agency for Research on Cancer. Globocan 2012: estimated cancer incidence, mortality and prevalence worldwide in 2012. [Internet] Lyon/France: IARC; 2014 [acesso em 10 dez 2016]. Disponível: http://globocan.iarc.fr/Default.aspx.

3. da Silva AF, Issi HB, Botene DZA, da Motta MGC. Cuidados paliativos em oncologia pediátrica: percepções, saberes e práticas na perspectiva da equipe multiprofissional. Rev. Gaúcha Enferm. [Internet] 2015;36(2) [acesso em 12 dez 2016]. Disponível: http://seer.ufrgs.br/index.php/RevistaGauchadeEnfermagem/article/view/46299.

4. dos Santos JL, Corral-Mulato S, Bueno SMV. Morte e luto: a importância da educação para o profissional de saúde. Arq. Cienc. Saúde UNIPAR. [Internet] 2014;18(3) [acesso em 10 dez 2016]. Disponível: http://revistas. unipar.br/index.php/saude/article/view/5196.

5. da Silva MM, de Santanda NGM, Santos MC, Cirilo JD, Barrocas DLR, Moreira MC. Cuidados paliativos na assistência de alta complexidade em oncologia: percepção de enfermeiros. Esc. Anna Nery. [Internet] 2015;19(3) [acesso em 01 set 2016]. Disponível: http://dx.doi.org/10.5935/1414-8145.20150061.

6. Calil AM, Prado C. Ensino de oncologia na formação do enfermeiro. Rev. bras. enferm. Brasília. [Internet] 2010;63(4) [acesso em 09 dez 2016]. Disponível: http://dx.doi.org/10.1590/S0034-71672010000400026.

7. Thuler LCS, Bergmann A, Ferreira SC. Ensino em atenção oncológica no Brasil: carências e oportunidades. Rev. bras. cancerol. [Internet] 2011;57(4) [acesso em 29 nov 2016]. Disponível: http://www.inca.gov.br/Rbc/n_57/v04/ pdf/02_artigo_ensino_atencao_oncologica_brasil_carencia_oportunidades.pdf.

8. Instituto Nacional de Câncer José Alencar Gomes da Silva (INCA). Coordenação Geral de Ações Estratégicas. Coordenação de Educação. Anke B, Thuler LCS, Canavarro SF, organizadores. Ensino em atenção oncológica no Brasil: carências e oportunidades [Internet] Rio de Janeiro: Inca; 2012 [acesso em 19 mai 2017]. Disponível: http:// bvsms.saude.gov.br/bvs/publicacoes/ensino_atencao_oncologica_brasil.pdf.

9. Henriques SHH, Silva VLS, Laus AM, Chaves LDP. Perfil profissional de enfermeiros atuantes em unidades de terapia intensiva de um hospital de ensino. Ciencia y Enferm. [Internet] 2013;19(3) [acesso em 04 mar 2016]. Disponível: http://dx.doi.org/10.4067/S0717-95532013000300006. 
10. Kobayashi RM, Leite MMJ. Desenvolvendo competências profissionais dos enfermeiros em serviço. Rev. bras. enferm. [Internet] 2010;63(2) [acesso em 03 mar 2016]. Disponível: http://dx.doi.org/10.1590/S003471672010000200012.

11. Camelo SHH. Competência profissional do enfermeiro para atuar em Unidades de Terapia Intensiva: uma revisão integrativa. Rev. Latino-Am. Enfermagem. [Internet] 2012;20(1) [acesso em 12 dez 2016]. Disponível: http:// dx.doi.org/10.1590/S0104-11692012000100025.

12. da Cruz FS, Rossato LG. Cuidados com o Paciente Oncológico em Tratamento Quimioterápico: o Conhecimento dos Enfermeiros da Estratégia Saúde da Família. Rev. bras. cancerol. [Internet] 2015;61(4) [acesso em 05 dez 2016]. Disponível: http://www1.inca.gov.br/rbc/n_61/v04/pdf/04-artigo-cuidados-com-o-paciente-oncologico-emtratamento-quimioterapico-o-conhecimento-dos-enfermeiros-da-estrategia-saude-da-familia.pdf.

13. Salimena AMO, Teixeira SR, Amorim TV, Paiva ACPC, Melo MCSC. O vivido dos enfermeiros no cuidado ao paciente oncológico. Cogitare Enferm. [Internet] 2013;18(1) [acesso em 03 nov 2016]. Disponível: http://dx.doi. org/10.5380/ce.v18i1.31320. 